\title{
Elección consensuada de una herramienta común de valoración sociosanitaria en la CAPV
}

\section{Elena Elosegui}

Servicio de Admisión y Documentación Clínica, Hospital Universitario Donostia, Osakidetza

<mariaelena.eloseguivallejo@osakidetza.net>

\section{Belen Elizalde}

Delegación Territorial de Gipuzkoa, Departamento de Salud, Gobierno Vasco

\section{Mari Jose Goñi}

Comarca Gipuzkoa de Atención Primaria, Osakidetza

\section{Olga Díaz de Durana}

Instituto Foral de Bienestar Social, Diputación Foral de Araba

\section{Adela Olascoaga}

Delegación Territorial de Bizkaia, Departamento de Salud, Gobierno Vasco

\section{Ander Zapiain}

Departamento de Política Social, Diputación Foral de Gipuzkoa

\section{Lourdes Zurbanobeaskoetxea}

Departamento de Acción Social. Diputación Foral de Bizkaia

\section{Olatz Albizua}

Servicio de Admisión y Documentación Clínica, Hospital Universitario Donostia, Osakidetza

\begin{abstract}
Artikulu honetan deskribatzen da Euskadin garatu den balorazio soziosanitariorako tresna komun eta adostu bat hautatzeko azterlana, eta horren bidez nahi da arlo sozial eta sanitarioak hizkuntza partekatu bat erabiltzea, eta horrek erraztea premia soziosanitarioak dituzten pertsonen arretaren jarraipen, integraltasun eta koordinazioa. Lehenengo aro batean, interRAI Contact Assessment identifikatu zen proposaturiko xedeak betetzeko baliagarrien den tresna gisara, eta egokitu zen euskal testuingurura. Horren ondoren, burutu zen froga pilotu bat balioztatze soziosanitariorako tresna hori osasun eta gizarte-zerbitzuetako arloetako diziplina desberdinetako 350 pertsonako lagina osatuz, eta esperientziaren ingurukoak hausnartu zituzten eztabaida-taldeetan. Azkenik, artikuluak biltzen ditu ebaluaturiko tresnaren egokitasunaren inguruan azterlanak azaldutako oinarrizko ondorioak eta Euskadiko oinarrizko arreta-mailetan egokitzeko betebeharreko baldintzak.
\end{abstract}

\section{GAKO-HITZAK:}

Arreta soziosanitarioa, ebaluazioa, gizartezerbitzuak, osasun-zerbitzuak, koordinazioa, tresna teknikoak.
Este artículo describe un estudio desarrollado a fin de escoger una herramienta común y consensuada de valoración sociosanitaria en Euskadi, que responda a la necesidad de avanzar en el establecimiento de un lenguaje compartido por los servicios sociales y sanitarios, y favorezca la continuidad, integralidad y coordinación de la atención que reciben las personas con necesidades sociosanitarias. En una primera fase, se identificó el interRAl Contact Assessment como la herramienta que mejor podría servir a los fines propuestos, y se adaptó al contexto vasco. Posteriormente, se realizó una prueba piloto donde un grupo multidisciplinar de profesionales de la salud y los servicios sociales aplicaron ese instrumento a la valoración sociosanitaria de $\mathbf{3 5 0}$ personas, y reflexionaron sobre la experiencia en grupos de discusión. Por último, el artículo recoge las principales conclusiones extraídas del estudio relativas a la idoneidad de la herramienta evaluada y a las condiciones para su adopción en los niveles básicos de atención sociosanitaria en Euskadi.

\section{Palabras Clave:}

Atención sociosanitaria, instrumentos técnicos, evaluación, coordinación, servicios sociales, servicios de salud. 


\section{Justificación, contextualización y principales objetivos}

\subsection{Necesidad de una herramienta común de valoración sociosanitaria}

Las personas con necesidad de cuidados sociales y sanitarios, así como sus familiares, aspiran a recibir una atención adecuadamente coordinada, continuada y de calidad. Las instituciones vascas son conscientes de esa demanda y comparten la visión de ofrecer una respuesta integral y centrada en la persona, orientada a garantizar el máximo nivel de bienestar, calidad de vida y autonomía; una respuesta que prioriza y facilita, siempre que es posible, su permanencia en el entorno social habitual. En coherencia con esta visión, uno de los objetivos básicos de los sistemas de bienestar debe ser garantizar la continuidad de los cuidados desde la perspectiva de la persona usuaria, mediante la provisión de un continuum de atención en el que la transición entre servicios -tanto entre diferentes niveles de especialización de un mismo sistema, como entre sistemas diferentes- se realiza con agilidad, sin interrupciones y de manera eficiente, y obtiene el máximo provecho de los recursos existentes.

En la Comunidad Autónoma Vasca (CAPV), las bases para la construcción de este espacio sociosanitario se establecieron hace ya una década, mediante un convenio de colaboración suscrito el 30 de enero de 2003 entre el Gobierno Vasco, las Diputaciones Forales de Álava, Bizkaia y Gipuzkoa, y Eudel Euskadiko Udalen Elkartea-Asociación de Municipios Vascos, para el desarrollo de la atención sociosanitaria en la CAPV (Gobierno Vasco et al., 2003) ${ }^{1}$. En dicho documento se opta, entre las tres grandes vías posibles ${ }^{2}$, por un modelo de coordinación de todas las instituciones competentes en la materia, basado en la armonización de las respectivas políticas, pero en el que se respeta la autonomía de gestión de cada una de las instituciones involucradas. Se trata, por tanto, de un modelo en el que se establece una fórmula de relación estructurada, que opera, sin embargo, desde las respectivas estructuras de los sistemas de atención existentes: trata de coordinar

${ }^{1}$ Hay numerosos antecedentes de acuerdos territoriales entre el Departamento de Salud del Gobierno Vasco y las diputaciones forales, pero el de 2003 constituye el primer acuerdo a escala autonómica $y$, por tanto, el hito inicial en la colaboración sociosanitaria en ese ámbito (Consejo Vasco de Atención Sociosanitaria, 2014).

${ }^{2}$ En el ámbito internacional, es habitual distinguir entre tres modelos de atención sociosanitaria según el grado de intensidad de la relación entre lo social y lo sanitario: a) la vinculación, donde los servicios buscan trabajar de forma coordinada, pero arbitrando soluciones ad hoc, para dar respuesta a casos concretos; b) la coordinación, donde se adoptan estrategias de colaboración más estructuradas, tratando de minimizar, paliar o evitar algunas de las barreras u obstáculos organizativos y administrativos existentes, pero respetando el esquema competencial y organizativo preexistente y la separación entre los diferentes ámbitos de atención; y c) la integración, donde existe una total imbricación de los ámbitos social y sanitario, en términos de competencias, organización de las redes de recursos, prestación de servicios y financiación, frecuentemente a través de la creación de un tercer sistema de bienestar (SIIS Centro de Documentación y Estudios, 2008). el uso de los diferentes servicios y prestaciones económicas asociadas a cada uno de los sistemas de atención; permite compartir e intercambiar información, aunque manteniendo en cada sector su propio sistema de datos; permite gestionar el traslado entre servicios; y permite determinar en cuál de los dos sectores recae la responsabilidad de coordinar la atención, atendiendo a la naturaleza y características de cada caso concreto.

Sea cual sea el modelo de coordinación elegido, garantizar la continuidad de la atención -objetivo principal del espacio sociosanitario y de todas las iniciativas de coordinación desarrolladas en este campo- exige en primer lugar, como requisito previo, establecer estrategias e instrumentos para compartir la información disponible sobre la situación de las personas atendidas, su contexto y trayectoria, sus expectativas, necesidades y perspectivas de evolución. En efecto, la información es el hilo conector entre las diferentes entidades y profesionales que intervienen en la prestación de la atención, y también entre diferentes episodios o contingencias que requieren una intervención sanitaria o social, de tal suerte que el trasvase de información entre profesionales de un servicio o entre diferentes servicios constituye un requisito previo esencial de la coordinación de la atención sociosanitaria (SIIS Centro de Documentación y Estudios, 2008).

Efectivamente, de acuerdo con Kodner y Spreeuwenberg (2002), prestar una atención bien articulada requiere adoptar un lenguaje común a través de criterios compartidos para el diagnóstico de necesidades, y el establecimiento de prácticas y estándares acordados entre ambos sistemas. Para lograrlo, pueden resultar de utilidad diferentes estrategias e instrumentos:

- Los criterios e instrumentos estandarizados de diagnóstico.

- Los procedimientos comunes de valoración integral.

- La planificación individual conjunta, los registros y expedientes comunes o compartidos.

- El seguimiento continuado.

- Los instrumentos comunes de apoyo a la toma de decisiones (guías de buena práctica y protocolos).

En la CAPV, se reconoce desde hace tiempo la necesidad de instrumentos comunes que contribuyan a la confluencia de lenguajes y a la construcción de este espacio compartido de comunicación para los y las profesionales de los ámbitos social y sanitario. Ya en 2003, el Consejo Vasco de Atención Sociosanitaria detectaba esta necesidad y constituía una comisión específica, cuyo objetivo era "proponer un sistema o instrumento técnico validado de medición de la dependencia, que será de uso común para toda la CAPV" (cit. en Urrutia, 2006: 21). Tras analizar catorce instrumentos de valoración -incluidos los que en aquel momento utilizaban las diputaciones forales, 
otros utilizados en el ámbito autonómico y los dos principales sistemas utilizados a escala internacional-, la Comisión propuso el instrumento RAI (20\%) y el sistema de clasificación RUG III como instrumento común de valoración en la CAPV, propuesta que fue aprobada por el Consejo en febrero de 2005 (Ibídem: 35-36).

A la hora de proponer la aplicación del RAI-RUG III, la Comisión tuvo en cuenta diversas características de la herramienta, que la convertían, a juicio de sus integrantes, en la elección más adecuada. Entre ellas, su extensa implantación, que facilitaría la comparación de la CAPV con otros países; el hecho de estar ampliamente validada, con un alto nivel de garantía científica; el de contar con un conjunto mínimo, aunque suficiente, de datos; y su capacidad para orientar a cada persona al recurso más idóneo en relación a sus necesidades.

A pesar de estar dotada de todas estas cualidades, la implantación del RAI-RUG III en el conjunto de la CAPV no pudo hacerse realidad. En parte, ello se debió a que en diciembre de 2006 -el mismo año en que comenzó a aplicarse el RAl- el Gobierno del Estado aprobó la Ley 39/2006, de 14 de diciembre, de Promoción de la Autonomía Personal y Atención a las Personas en Situación de Dependencia, con la consiguiente introducción del Baremo de Valoración de la Dependencia (BVD) y la Escala de Valoración Específica de Dependencia para Personas Menores de Tres Años (EVE) como instrumentos de valoración obligatorios para todas las personas que quisieran acceder a las prestaciones del Sistema para la Autonomía y Atención a la Dependencia (SAAD). Ello distorsionó el proceso de aplicación común previsto por el Consejo Vasco de Atención Sociosanitaria, de forma que, actualmente, las Diputaciones de Bizkaia y Gipuzkoa utilizan ambas herramientas (RAI-RUG III y BVD), mientras que la de Álava ha optado por utilizar únicamente el BVD.

A esto se le suma el hecho de que en el ámbito sanitario tampoco existe un único instrumento de uso común en los tres territorios históricos que valore las necesidades de atención sociosanitaria: en Gipuzkoa, se aplica la Escala de Valoración Sanitaria (EVS); y en Bizkaia, la Valoración Geriátrica Básica (VGB); mientras que en Álava, no existe ninguna herramienta que se aplique de forma generalizada.

Así, actualmente, el único instrumento de uso común en los tres territorios históricos para valorar a la población diana de los servicios sociosanitarios es el Baremo de Valoración de la Dependencia (BVD), introducido como puerta de acceso a los servicios contemplados en la llamada Ley de Dependencia. Con todo, es importante destacar que este instrumento sólo valora el desempeño de las actividades básicas e instrumentales de la vida diaria, por lo que no ofrece la información necesaria para orientar a los y las profesionales en lo referente al acceso a servicios de carácter sociosanitario. Todo ello conduce a que los ingresos de la población diana en recursos de media estancia hospitalaria y en las unidades residenciales sociosanitarias no siempre se realicen de forma suficientemente equitativa ni objetiva, y pone de manifiesto la necesidad de avanzar en el uso de una herramienta de valoración común. Del mismo modo, la inexistencia de un lenguaje común y compartido entre los sistemas sanitario y social provoca otras distorsiones de importancia, como la repetición en la recogida de datos, la proliferación de valoraciones o la alimentación paralela de sistemas informáticos, que dificultan gravemente la continuidad asistencial.

Para superar esta situación y poder ofrecer la atención sociosanitaria de calidad que la sociedad vasca demanda, es necesaria, por lo tanto, la adopción consensuada de un instrumento común de valoración sociosanitaria. Este instrumento:

- Serviría como lenguaje común para los y las profesionales de los sistemas de servicios sociales y de salud, permitiéndoles elaborar sus intervenciones basándose en una valoración objetiva y compartida de la situación de la persona.

- Facilitaría una rápida derivación de las personas con necesidades complejas hacia los dispositivos sociales, sanitarios y sociosanitarios pertinentes.

- Permitiría detectar y poner de manifiesto situaciones en las que existe un vacío en el encaje entre el sistema sanitario y social, y otras en las que se duplican o solapan servicios e intervenciones.

- Constituiría el embrión de una historia sociosanitaria individual en Euskadi.

\subsection{La oportunidad para el cambio}

A pesar de las dificultades y obstáculos que se han ido encontrando en el camino, el momento actual se presenta como una gran oportunidad para avanzar hacia la visión descrita y, más concretamente, hacia el uso de una herramienta común de valoración en el ámbito sociosanitario.

En primer lugar, las líneas estratégicas adoptadas por el Consejo Vasco de Atención Sociosanitaria en 2013 (Consejo Vasco de Atención Sociosanitaria, 2014) ponen medios para construir un lenguaje común para los y las profesionales del ámbito social y sanitario en la CAPV, al aludir a la necesidad de implantar un sistema de información y comunicación sociosanitario, en el que tendrá un papel central la historia sociosanitaria. Ésta contará con un conjunto mínimo de datos, obtenidos por medio de un procedimiento común de valoración, en el que se aplicará un instrumento consensuado para su uso en ambos sistemas, sea cual sea el territorio histórico y nivel asistencial desde el que se acceda a los recursos.

Por otra parte, la construcción de un lenguaje común con el que estructurar y articular el espacio sociosanitario, en tanto que objetivo estratégico, se ve reforzado por su inclusión en los respectivos planes sectoriales de salud y de servicios sociales: 
- Así, el nuevo Plan de Salud de Euskadi (20132020) [Departamento de Salud, 2013] menciona, entre las acciones dirigidas a mejorar la atención sociosanitaria prestada a la población mayor, el desarrollo y la implantación de la historia sociosanitaria, así como de un instrumento multidisciplinar y multisectorial de valoración geriátrica integral, enfocado a la prevención, detección y manejo de problemas para personas mayores de 75 años, o personas menores de esta edad frágiles, con pluripatología o déficit cognitivo. De cara a avanzar en este camino, prevé además que, para 2015, todo el ámbito sociosanitario disponga de acceso a la historia clínica. Como primer paso en esta dirección, los centros residenciales para personas mayores tendrán acceso a Osabide Global a partir de otoño de 2014 (Gobierno Vasco, 2013).

- En el documento de Líneas estratégicas y planes de acción 2013-2016 (Osakidetza, 2013), por su parte, se plantea el objetivo de ofrecer una respuesta integrada a nuevos retos como la vejez, la cronicidad y la dependencia. Para ello, se prevé, en primer lugar, extender el modelo de organizaciones sanitarias integradas (OSI), que son la agrupación de los centros de atención primaria y su hospital de referencia en una demarcación geográfica definida, y en las que, progresivamente, se irán integrando las estructuras de salud pública y del ámbito sociosanitario. La puesta en funcionamiento de estas estructuras integradas exigirá la utilización de instrumentos comunes de valoración, lo que constituye una oportunidad para implantarlos.

- También el Plan Estratégico de Servicios Sociales de la CAPV (Gobierno Vasco, 2011) señala, al realizar el diagnóstico de los elementos estructurales que se consideran prioritarios de cara a la consolidación del sistema, la necesidad de arbitrar nuevas fórmulas en el ámbito de la organización de servicios, y de fomentar el trabajo interdisciplinar orientado a la valoración conjunta de necesidades $y$ al diseño de paquetes de cuidados sociales y sanitarios bien articulados. Así, dentro del objetivo estratégico 6.1., referido a la culminación del espacio sociosanitario, se plantea la elaboración de instrumentos y protocolos conjuntos de colaboración sociosanitaria.

\subsection{Objetivos}

En este contexto, en el que se aprecian importantes sinergias para la búsqueda y la construcción de un lenguaje común para el ámbito social y sanitario, Etorbizi, la Fundación Vasca para la Innovación instrumento de valoración que fuera la puerta de entrada única al sistema sociosanitario. En concreto, los objetivos que guiaron la realización de ese proyecto fueron tres:

- Seleccionar un instrumento común de valoración integral para la planificación de cuidados sociosanitarios de la población diana que permita el acceso equitativo a todos los recursos sociosanitarios y oriente la ruta sociosanitaria.

- Consensuar la selección de este instrumento entre agentes institucionales y no institucionales.

- Conocer si, una vez consensuado, el instrumento elegido es realmente válido a la hora de aplicarlo en el contexto de la CAPV.

En ese sentido, desde un principio se partió de la base de que el instrumento en cuestión había de ser consensuado por las instituciones implicadas y había de permitir:

- prevenir para potenciar la autonomía,

- valorar integralmente a la persona,

- orientar hacia el recurso más idóneo, y

- ubicar elementos tales como discapacidad, dependencia, cronicidad, convalecencia o terminalidad.

En lo que se refiere a la población diana, se consideró desde un primer momento que la aplicación del instrumento iría dirigida a personas con los siguientes perfiles o características:

- mayores en situación de dependencia,

- con discapacidad,

- con enfermedades somáticas crónicas 0 invalidantes,

- con enfermedades terminales, 0

- convalecientes de enfermedades (alta hospitalaria sin autonomía para el autocuidado).

El presente artículo describe ese proyecto de investigación, que fue promovido por la Fundación Vasca de Innovación e Investigación Sanitarias (BIOEF) y, como se ha señalado, contó con la financiación de EtorbiziFundación Vasca para la Innovación Sociosanitaria, en el marco de su convocatoria de ayudas a proyectos de investigación sociosanitaria correspondiente a 2011. Tras aceptarse una prórroga del proyecto por la ampliación de los objetivos iniciales propuestos, el proyecto terminó con la celebración de unas jornadas técnicas el 11 de septiembre de 2014 en el Centro Carlos Santamaría de Donostia-San Sebastián (Elósegui et al., 2014). 


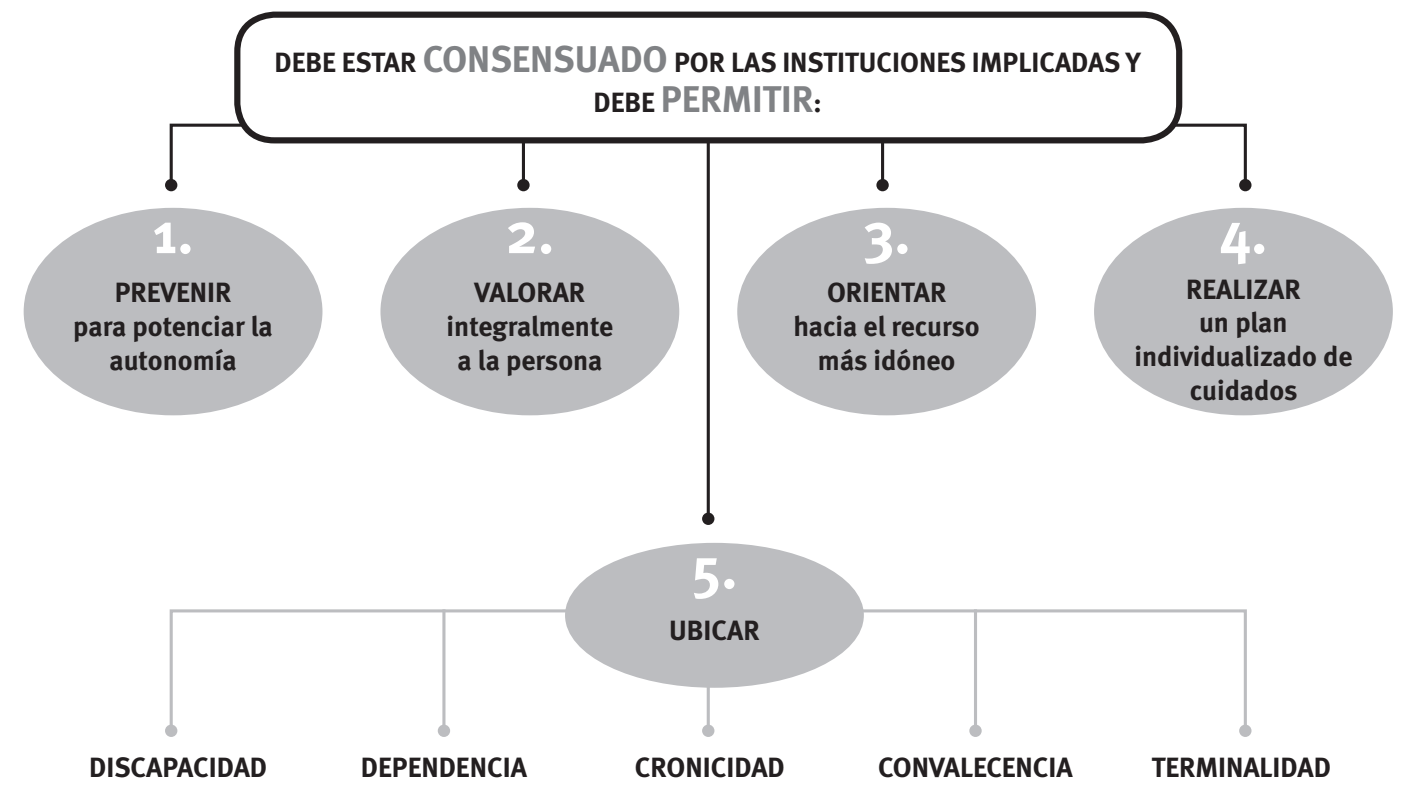

Fuente: Elaboración propia.

\section{Resultados de la investigación}

\subsection{Elección consensuada de un instrumento de valoración sociosanitaria: revisión bibliográfica, selección y descripción del instrumento}

La primera fase de la investigación consistió, como se ha dicho, en seleccionar un instrumento de valoración sociosanitaria susceptible de ser utilizado en el ámbito social y en el sanitario, en el marco del espacio sociosanitario de la CAPV. La revisión bibliográfica y el trabajo desarrollado por el grupo de expertos dieron como resultado la elección del interRAI Contact Assessment (interRAI CA).

\subsubsection{Revisión bibliográfica: objetivos y principales resultados}

La revisión bibliográfica realizada en el marco del proyecto se diseñó en función de cuatro objetivos: a) identificar instrumentos de valoración; b) seleccionar instrumentos válidos; c) analizar las propiedades psicométricas de los instrumentos seleccionados; y d) elegir escalas que cumplieran con las características exigidas.

Idealmente, el instrumento adecuado debía ser multidimensional, es decir, incluir diferentes dimensiones de valoración como actividades de la vida diaria (AVD), capacidad cognitiva, estado emocional, morbilidad, necesidades de cuidados de enfermería, o apoyo social; tener evidencia científica demostrada sobre su validez, fiabilidad y sensibilidad al cambio en la medida de la discapacidad; y disponer de una traducción y validación en inglés o castellano.

En función de estos criterios, de entre más de 150 escalas o instrumentos encontrados durante la revisión bibliográfica se destacaron básicamente dos alternativas:

- interRAl: sistema de evaluación ampliamente extendido en EE.UU., Canadá y Australia, y cada vez con mayor implantación en Europa. Inicialmente, en 1990, se diseñó para su aplicación en centros de larga y media estancia, si bien de forma progresiva se han ido creando módulos para su uso en otros contextos (urgencias, domicilio). Hoy día dispone de once módulos, como atención domiciliaria, hospital de agudos, cuidados postagudos, cuidados paliativos, salud mental, salud mental comunitaria o discapacidad intelectual. La última versión, interRAI 3.0 (2010), ha implementado la tecnología necesaria para permitir la transferencia de datos estandarizados entre diferentes niveles de asistencia sociosanitaria, convirtiéndolo en el único sistema integrado y definido, por ello, como ‘de tercera generación'.

De acuerdo con la revisión realizada, el sistema interRAI 3.0 ofrece ventajas indiscutibles frente a otras alternativas de cara a su uso para valorar las necesidades sociosanitarias. Además de permitir una evaluación integral individual tanto de la salud, como de la función y del entorno, ha implementado 
tecnologías que permiten la continuidad de cuidados entre diferentes servicios sociosanitarios y la medida longitudinal de la dependencia tanto en el ámbito intra como extramural. Su principal inconveniente es el considerable consumo de recursos que requiere en cuanto a tiempo para las evaluaciones, entrenamiento del personal y recursos tecnológicos. A modo de conclusión, dada la importancia de utilizar un sistema de valoración estándar y uniforme, y con amplia evidencia científica respecto a sus propiedades de medida, la investigación recomendaba analizar detenidamente, mediante estudios piloto, las características prácticas en la aplicación del interRAl en los diferentes ámbitos y por los diferentes agentes implicados en el proceso.

- WHODAS-II (World Health Organization Disability Assessment Schedule 2.0, UK 1998/2001): escala de discapacidad diseñada bajo el marco conceptual de la Clasificación Internacional del Funcionamiento, la Discapacidad y la Salud (CIF). Ha sido ampliamente validada en múltiples países e idiomas, también en España, en población hospitalaria y de rehabilitación. Entre las escalas de discapacidad revisadas, el WHODAS-II puede ser una opción válida por su difusión internacional, su integración en el modelo de discapacidad propuesto por la OMS, y por existir amplia evidencia científica de su validez y fiabilidad en la práctica clínica.

\subsubsection{Elección del interRAI CA para su uso en el nivel básico de atención sociosanitaria}

A partir de las conclusiones y propuestas derivadas de la revisión bibliográfica, el grupo de investigación optó por centrarse en la familia de instrumentos interRAI (Home Care y Screener Plus), al ser una de las herramientas que ya se utiliza para evaluar las necesidades de atención social y sanitaria en la CAPV. Asimismo, consideraba que esta familia de instrumentos permite alcanzar la multifuncionalidad planteada en el objetivo general de proyecto. Profundizando en la familia interRAl, se constató además que presenta una gama de herramientas que van desde el cribado inicial a la valoración integral, lo que permite el uso compartido de un conjunto básico de datos en los diferentes sistemas asistenciales, así como en los niveles básico y especializado de cada sistema.

Tras varias sesiones de discusión con los grupos de expertos establecidos al efecto, entre los cuales se incluyó también a un experto en instrumentos de la familia interRAl, el grupo de investigación determinó proponer la adaptación y utilización en la CAPV del interRAI Contact Assessment (interRAI CA) como instrumento básico de valoración sociosanitaria.

\subsubsection{Características básicas del interRAl CA}

El interRAI Contact Assessment es un instrumento de cribado o estratificación poblacional, diseñado para su uso tanto en la comunidad como en el ámbito hospitalario (ingresados y servicios de urgencias). Está formado por dos módulos: el módulo comuni$\mathrm{dad} /$ hospital y el módulo de servicio de urgencias. El primero está pensado como instrumento de evaluación de la necesidad de servicios en domicilio a largo plazo para las personas en la comunidad o en el momento del alta desde recursos hospitalarios (incluido el servicio de urgencias). Por su parte, el módulo del servicio de urgencias está diseñado para apoyar las decisiones al alta, agilizando los procesos de derivación bien a otros niveles hospitalarios o bien a domicilio, permitiendo, en este caso, identificar los servicios posteriores al alta que garanticen la continuidad asistencial. Hay que cumplimentar los dos instrumentos de manera electrónica para asegurar que las personas encargadas de la evaluación reciben los resultados de los algoritmos clínicos e indicadores del interRAI CA de manera instantánea. La diferencia entre ambos se centra en el problema derivado del tiempo y la información necesarios en los diferentes dispositivos. Los dos módulos comparten la misma lógica clínica, ítems e indicadores clínicos. La compatibilidad de los elementos de los dos módulos promueve un abordaje centrado en las personas e incrementa la capacidad de transición entre la atención aguda y los servicios comunitarios.

El primer módulo se articula en torno a cinco secciones:

- La sección A documenta la información demográfica y los motivos por los que se solicita la derivación.

- La sección B contiene información sobre los detalles de la derivación (tipo de servicio que parece que se necesita) y la organización familiar de la persona.

- La sección C contiene los ítems que se utilizan en un algoritmo de decisión, que diferencia a las personas que no necesitan una valoración más exhaustiva de aquellas que requieren una valoración más amplia posterior.

- La sección D contiene el resto de los ítems necesarios para los algoritmos de decisión clínicos, así como la información clínica adicional sobre las personas que requerirán servicios de corta duración.

- La sección E registra la información resumida de los algoritmos y de la necesidad de servicios:

- El algoritmo de Urgencia de la Valoración: en una escala de 1 a 6 , las puntuaciones más elevadas implican una mayor urgencia en la realización de una evaluación general o por áreas más exhaustiva.

- El algoritmo de Necesidad de Servicios: en una escala de 1 a 4, las puntuaciones más elevadas suponen una mayor urgencia en el acceso a servicios.

- El algoritmo de Rehabilitación: en una escala de 1 a 5 , los valores más elevados indican una mayor probabilidad de necesidad de servicios de rehabilitación/recapacitación. 
Además de los algoritmos descritos, el Contact Assessment elabora un Índice de Dependencia (Self-Reliance Index). Se trata de un indicador descriptivo que diferencia a las personas autónomas de aquellas que no lo son en relación con la capacidad para la ejecución adecuada de AVD y a la capacidad cognitiva para tomar decisiones.

El segundo módulo es un instrumento de cribado estandarizado para uso clínico. Se ha diseñado para recoger y registrar la información necesaria en los servicios de urgencia. Está articulado en tres secciones -cribado preliminar (F), evaluación clínica (G) y resumen $(\mathrm{H})$ - y ofrece los siguientes algoritmos:

- El Índice de Dependencia, al que ya se ha hecho referencia.

- El algoritmo de Valoración de la Urgencia se utiliza para identificar la necesidad y urgencia de realizar una evaluación general o por áreas más exhaustiva, bien en comunidad, bien en servicios especializados, y permite predecir la probabilidad de ingresar en el hospital o en servicios alternativos de cuidados.

- El algoritmo de Necesidad de Servicio se utiliza para identificar a las personas que puedan tener necesidad de servicios comunitarios y la urgencia del acceso a aquéllos.

El interRAI CA no sustituye a una valoración integral: registra, en el momento del contacto inicial o en revisiones periódicas posteriores, la información esencial necesaria para determinar la situación de autonomía de las personas (identificando tanto los aspectos positivos como las necesidades y problemas de la persona), la necesidad de realizar una valoración general o por áreas más amplia, la necesidad de servicios de apoyo en domicilio o la necesidad de servicios especializados, por ejemplo, de rehabilitación o recapacitación funcional. Como se ha señalado, constituye la puerta de entrada al espacio sociosanitario con una filosofía de trasdisciplinariedad, que posteriormente permita una intervención interdisciplinar integrada.

\subsubsection{Razonas básicas para la elección del interRAI CA como herramienta común de valoración sociosanitaria}

Como se ha apuntado anteriormente, la revisión bibliográfica y el posterior trabajo de análisis evidenció que, a pesar de existir una gran diversidad de herramientas de valoración de la dependencia o discapacidad (se encontraron más de 150 escalas/instrumentos), sólo dos parecían capaces de responder a las necesidades plateadas: el paquete de instrumentos de la denominada familia RAI, y el WHODASII. Sin embargo, el equipo de investigación llegó a la conclusión de que sólo la familia RAI respondía a las necesidades exigidas, especialmente en lo referido a la interoperabilidad entre sistemas, en la medida en que ha implementado la tecnología necesaria para permitir la transferencia de datos estandarizados entre diferentes niveles de asistencia sociosanitaria. Ello lo convierte en el único sistema integrado y permite considerarlo como ‘de tercera generación’.

La selección de la familia RAI permite optar por una gran diversidad de herramientas que se pueden utilizar en diferentes niveles asistenciales. Se considera, en ese sentido, que es la herramienta idónea para garantizar una adecuada valoración de las necesidades de los usuarios, independientemente de dónde se preste la atención. El paquete de instrumentos está

Figura 2. Instrumentos de valoración a utilizar en organizaciones sociosanitarias

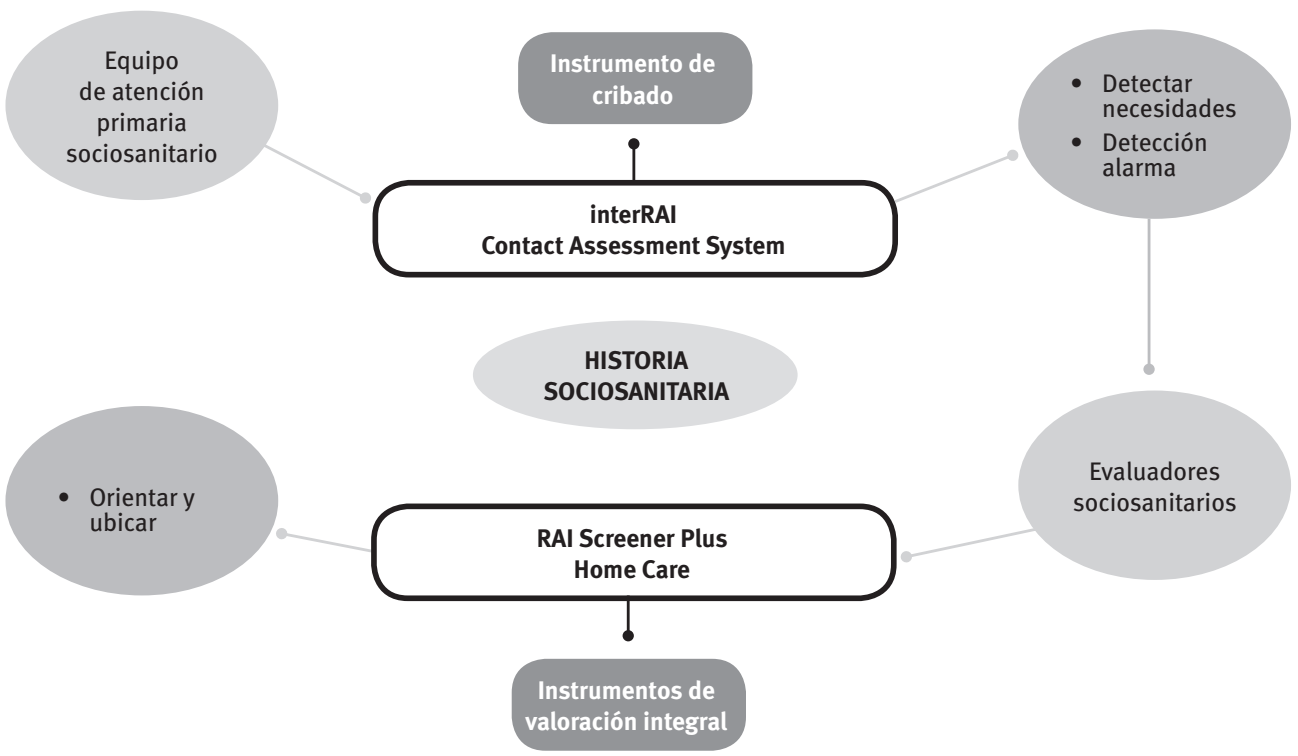

Fuente: Elaboración propia. 
construido con una base común de ítems de evaluación que se consideran relevantes en todos los dispositivos. Estos ítems comunes tienen definiciones, tiempos de observación y puntuación idénticas, pero en cada una de las herramientas, se añaden ítems específicos para determinadas poblaciones o dispositivos.

Esta decisión es coincidente con la tomada en su día por el Consejo Vasco de Atención Sociosanitaria, que, como se ha visto, determinó en 2003 la necesidad de dotar a la CAPV de un modelo unificado de valoración de la dependencia y en 2005 propuso utilizar para tal fin el sistema RAI (20\%) y el clasificador RUG III (Urrutia, 2006). A juicio del equipo de investigación, la novedad que aporta este proyecto respecto a la decisión anterior se basa en la estrategia de implantación. En nuestro caso, se establece como puerta de entrada a la atención sociosanitaria un instrumento sencillo de la familia RAI, el interRAI CA, para uso de los y las profesionales de atención primaria sociosanitaria (profesionales de la medicina, la enfermería y el trabajo social), una herramienta que permite proponer un cribado o valoración compartiendo la misma información. Desde ahí, se podrá acceder a la historia sociosanitaria o determinar si es necesario pasar a una valoración integral con el fin de orientar o adjudicar otros recursos o servicios, tal y como se ve en la Figura 2, lo que permite la interoperabilidad entre los diferentes niveles asistenciales.

En ese sentido, el interRAI CA, como herramienta básica, permite utilizar otras herramientas de valoración de carácter más especializado que no necesariamente han de pertenecer a la familia del RAl, aunque sí han de compartir con ella una serie de datos fundamentales que, junto con una aplicación informática común, permitan la interoperabilidad en el sistema. En la Figura 2 pueden observarse las diferentes herramientas y su posible utilización según los objetivos que se pretenden obtener.

\subsection{Adaptación del instrumento}

Una vez seleccionado el instrumento, el equipo investigador determinó la necesidad de traducirlo y adaptarlo para poder utilizarlo en nuestro medio. Ese proceso -en el que se colaboró con dos entidades, interRAl y RAlsoft, mediante bajo la fórmula de proyectos de investigación- se realizó en dos fases:

- Validación del interRAl en los dos idiomas oficiales de la CAPV, lo cual incluyó:

a. Traducción del instrumento interRAI CA y del manual de uso desde su versión original.

b. Validación de la traducción con expertos del equipo en el uso del interRAI CA.

c. Retrotraducción al inglés.

d. Aceptación de la adaptación realizada por Sergio Ariño, delegado de interRAl en España.

- Adaptación de una aplicación informática de la empresa RAlsoft, diseñada para utilizar el interRA CA en el municipio finlandés de Kokkola, que per- mita elaborar y administrar automáticamente los algoritmos de resultados. Esta aplicación, además de dar soporte al instrumento mediante un formulario de datos, permite conocer los resultados de aquél inmediatamente, pues genera una serie de informes que facilitan el trabajo diario de los y las profesionales de primera línea y permite explotar los datos a diferentes escalas (usuario individual, organización y sistema), de manera que se genera información útil tanto para la intervención como para la planificación y la gestión.

Incluso considerando la conveniencia de introducir algunas mejoras en la aplicación informática y el propio instrumento, disponer ya de una versión traducida y adaptada del interRAI CA, que ha sido validada por la organización que lo ha creado, así como de un programa informático en castellano y euskera, amigable y fácil de usar, que permite administrar ese instrumento de forma sencilla debe considerarse una primera aportación de interés del proyecto.

\subsection{Prueba piloto del instrumento}

Con el cuestionario y manual de uso traducidos, adaptados y validados y la aplicación informática a punto, se diseñó la prueba piloto del interRAI CA en nuestro medio, cuyos objetivos eran básicamente dos:

- Valorar si los y las profesionales del sector social y el de salud de nuestro contexto asistencial consideran interRAI CA como una herramienta útil de cribado para detectar necesidades sociosanitarias y monitorizar las variaciones de éstas que puedan requerir cambios en el plan de atención.

- Valorar la utilidad de interRAI CA como lenguaje común de comunicación entre los y las profesionales del ámbito sanitario y del ámbito social, de tal forma que sirva como puerta de entrada a la atención sociosanitaria y favorezca intervenciones conjuntas entre ambos sistemas.

Para realizar la prueba piloto, se seleccionó a un grupo de 36 profesionales de los tres territorios de la CAPV, combinando varias características:

- Perfil profesional: eran enfermeras, médicos/as o trabajadoras sociales.

- Ámbito de trabajo: procedían tanto del ámbito de la salud como del de los servicios sociales, $y$, en ambos casos, tanto de centros de atención primaria como de secundaria o especializada. En el caso del sistema sanitario, trabajaban en consejo sanitario, atención primaria, hospitales de agudos o de media estancia, o en servicios de urgencias hospitalarios. En el caso del sistema de servicios sociales, provenían básicamente de los servicios sociales de base.

El Cuadro 1 describe la procedencia y las características de las personas que participaron en la realización de las valoraciones en esta experiencia piloto. 


\begin{tabular}{|c|c|c|c|}
\hline & Araba & Bizkaia & Gipuzkoa \\
\hline Ámbito comunitario & $\begin{array}{l}\text { - Dos trabajadoras sociales de los } \\
\text { servicios sociales de base de base } \\
\text { de Vitoria-Gasteiz. } \\
\text { - Tres trabajadoras sociales de los } \\
\text { servicios sociales de la Cuadrilla } \\
\text { de Añana. } \\
\text { - Dos enfermeras de consejo } \\
\text { sanitario. } \\
\text { - Dos enfermeras de atención prima- } \\
\text { ria de Vitoria-Gasteiz. } \\
\text { - Una enfermera de atención } \\
\text { primaria del Centro de Salud de } \\
\text { Ribavellosa (Añana). } \\
\text { - Un médico de atención primaria de } \\
\text { Vitoria-Gasteiz. }\end{array}$ & $\begin{array}{l}\text { - Tres trabajadoras sociales de los } \\
\text { servicios sociales de base de Irala } \\
\text { y San Francisco (Ayuntamiento de } \\
\text { Bilbao) y una de la mancomunidad } \\
\text { de Mungialdea. } \\
\text { - Dos enfermeras de consejo } \\
\text { sanitario. } \\
\text { - Una enfermera gestora de casos } \\
\text { de la OSI Bilbao-Basurto y una } \\
\text { enfermera gestora de casos de la } \\
\text { comarca Uribe. } \\
\text { - Una médica de atención primaria } \\
\text { de la OSI Bilbao-Basurto (Centro de } \\
\text { Salud de Rekalde). } \\
\text { - Una médica responsable sociosa- } \\
\text { nitaria de la Diputación Foral de } \\
\text { Bizkaia. }\end{array}$ & $\begin{array}{l}\text { Dos trabajadoras sociales de los } \\
\text { servicios sociales de base de } \\
\text { Donostia-San Sebastián y una de } \\
\text { Irun. } \\
\text { - Dos enfermeras de consejo } \\
\text { sanitario. } \\
\text { - Una enfermera de atención prima- } \\
\text { ria de la comarca Donostia (Centro } \\
\text { de Salud de Prim) y una de la OSI } \\
\text { Bidasoa (Centro de Salud de Irún } \\
\text { Centro). } \\
\text { - Una médica de atención primaria } \\
\text { de la Comarca Donostia (Centro de } \\
\text { Salud de Pasai San Pedro). }\end{array}$ \\
\hline Ámbito hospitalario & $\begin{array}{l}\text { - Una trabajadora social del Servicio } \\
\text { Social de Urgencias del Hospital } \\
\text { Universitario de Álava. }\end{array}$ & $\begin{array}{l}\text { - Una enfermera de enlace del Hos- } \\
\text { pital Santa Marina. } \\
\text { - Un trabajador social del Hospital } \\
\text { Basurto. } \\
\text { - Una médica de urgencias del } \\
\text { Hospital de Cruces. }\end{array}$ & $\begin{array}{l}\text { - Dos enfermeras y una médica del } \\
\text { Hospital Donostia. } \\
\text { - Una enfermera de la Clínica La } \\
\text { Asunción de Tolosa. } \\
\text { - Una trabajadora social y una enfer- } \\
\text { mera del Hospital Matia. }\end{array}$ \\
\hline
\end{tabular}

OSI: organización sanitaria integrada.

Fuente: Elaboración propia.

Todos/as los/as profesionales participantes realizaron previamente un curso de adiestramiento en la utilización de la herramienta interRAI CA y la aplicación informática RAlsoft. El curso tuvo una duración de quince horas y, con el fin de facilitar la asistencia, se realizó una sesión por territorio, entre los meses de enero y febrero de 2014 , en horario de 8 a 14 horas. Las sesiones se organizaron de manera que a la formación sobre las partes específicas de los módulos comunitario y hospitalario del cuestionario acudieran los y las profesionales que los iban a utilizar específicamente, coincidiendo todos/as los/as profesionales en las partes comunes del cuestionario. El curso fue impartido por profesionales del Servicio de Evaluación y Orientación de Matia Fundazioa, con gran experiencia en sistemas de evaluación sociosanitarios y en la actividad docente.

La distribución de los/as participantes según territorio histórico y perfil profesional puede verse en la Tabla 1. La mitad fueron enfermeras; un $36 \%$, trabajadoras sociales; y un $14 \%$, médicos/as. Cada profesional cumplimentó 10 cuestionarios, con lo que se cumplimentaron un total de 360 .
Las personas participantes en el curso rellenaron una ficha de evaluación. Para la totalidad de ellas, la opinión global del curso fue 'buena' o 'muy buena'. El 96,3\% consideró que se habían cumplido los objetivos del curso de una forma 'buena' o 'muy buena'. Además, el $80 \%$ señaló que la utilidad práctica de lo aprendido era 'buena' o 'muy buena'. Al comparar todos los aspectos del curso, el territorio que mejor calificó los ítems fue Gipuzkoa.

En lo que se refiere a las personas a las que se aplicó el instrumento, se seleccionaron cuatro muestras: tres estaban formadas por personas atendidas en el ámbito comunitario, y la cuarta, por personas atendidas en el ámbito hospitalario, según los criterios de inclusión que se detallan en el Cuadro 2. No se incluyó a personas domiciliadas en residencias ni en pisos tutelados. La edad media de las 336 personas cuyos datos fueron finalmente registrados era de 78,6 años, y el $64 \%$ eran mujeres.

En cuanto a la administración de la herramienta, las secciones del cuestionario interRAI CA utilizadas por cada profesional dependieron del entorno de

Tabla 1. Participantes en el curso según perfiles profesionales

\begin{tabular}{|l|c|c|c|c|}
\cline { 2 - 5 } \multicolumn{1}{c|}{} & Enfermeras & Médicos/as & $\begin{array}{c}\text { Trabajadoras } \\
\text { sociales }\end{array}$ & Total \\
\hline Gipuzkoa & 8 & 2 & 4 & 14 \\
\hline Bizkaia & 5 & 2 & 5 & 12 \\
\hline Araba & 5 & 1 & 4 & 36 \\
\hline CAPV & 18 & 5 & 13 & 10 \\
\hline
\end{tabular}

Fuente: Elaboración propia. 


\begin{tabular}{|l|c|c|l|}
\hline Cuadro 2. Composición y características de las cuatro muestras a las que se aplicó la herramienta \\
\hline Muestra & $\begin{array}{c}\text { Tamaño de la } \\
\text { muestra* }\end{array}$ & \multicolumn{1}{|c|}{ Criterios de inclusión } & \multicolumn{1}{|c|}{$\begin{array}{c}\text { Método de aplicación del } \\
\text { cuestionario }\end{array}$} \\
\hline $\begin{array}{l}\text { Servicios sociales de base } \\
\text { municipales }\end{array}$ & 96 & $\begin{array}{l}\text { - Personas mayores de } 64 \text { años que acudieron a solicitar } \\
\text { atención de los servicios municipales de base partici- } \\
\text { pantes en el estudio. }\end{array}$ & $\begin{array}{l}\text { Presencial, por trabajadoras } \\
\text { sociales de base. }\end{array}$ \\
\hline Consejo sanitario & 58 & $\begin{array}{l}\text { - Personas mayores de } 64 \text { años usuarias de teleasisten- } \\
\text { cia (betiON) que llamaron al servicio y cuya demanda } \\
\text { se identificó como de contenido sanitario, por lo que } \\
\text { fue transferida a las enfermeras de consejo sanitario. }\end{array}$ & $\begin{array}{l}\text { Telefónica, por enfermeras de } \\
\text { consejo sanitario. }\end{array}$ \\
\hline $\begin{array}{l}\text { Atención primaria de } \\
\text { Osakidetza }\end{array}$ & 92 & $\begin{array}{l}\text { - Personas mayores de } 64 \text { años señaladas como pluripa- } \\
\text { tológicas en la historia clínica Osabide. }\end{array}$ & $\begin{array}{l}\text { Presencial, en el centro } \\
\text { de salud, por médico/a o } \\
\text { enfermera }\end{array}$ \\
\hline Servicios hospitalarios & 90 & $\begin{array}{l}\text { - Personas mayores de } 64 \text { años que acudieron al servicio } \\
\text { de urgencias y tenían indicación de retorno a domicilio } \\
\text { (con apoyos o sin ellos). } \\
\text { Personas mayores de } 64 \text { años pluripatológicas ingre- } \\
\text { sadas en una unidad de enfermería de agudos y en dos } \\
\text { unidades de media estancia. }\end{array}$ & $\begin{array}{l}\text { Presencial, en el centro } \\
\text { hospitalario, por médico/a o } \\
\text { enfermera. }\end{array}$ \\
\hline
\end{tabular}

*Se recogen únicamente los 336 casos incluidos finalmente en la aplicación informática.

Fuente: Elaboración propia.

aplicación. Concretamente, los y las profesionales de ámbito comunitario utilizaron los módulos del cuestionario de aplicación en la comunidad (secciones $A, B, C, D$ y E), mientras que los y las profesionales de urgencias hospitalarias cumplimentaron los módulos correspondientes (secciones A, F, G y H). Para la recogida de la información, se utilizó la aplicación informática previamente desarrollada, que, además de registrar las respuestas obtenidas, ofrece en tiempo real los algoritmos o resultados de la valoración. De esta forma, las encuestadoras podían cumplimentar el cuestionario y disponer de inmediato de los resultados. La aplicación había sido ya descrita en la sesión de formación y se aportó un número de teléfono al que recurrir en el caso de que surgieran dificultades en el transcurso de la aplicación del cuestionario.

La Figura 3 describe de forma sintética las fases del proceso seguido en la experiencia piloto de aplicación de la herramienta.

\section{Figura 3. Fases realizadas para la valoración del instrumento interRAI CA en la CAPV}

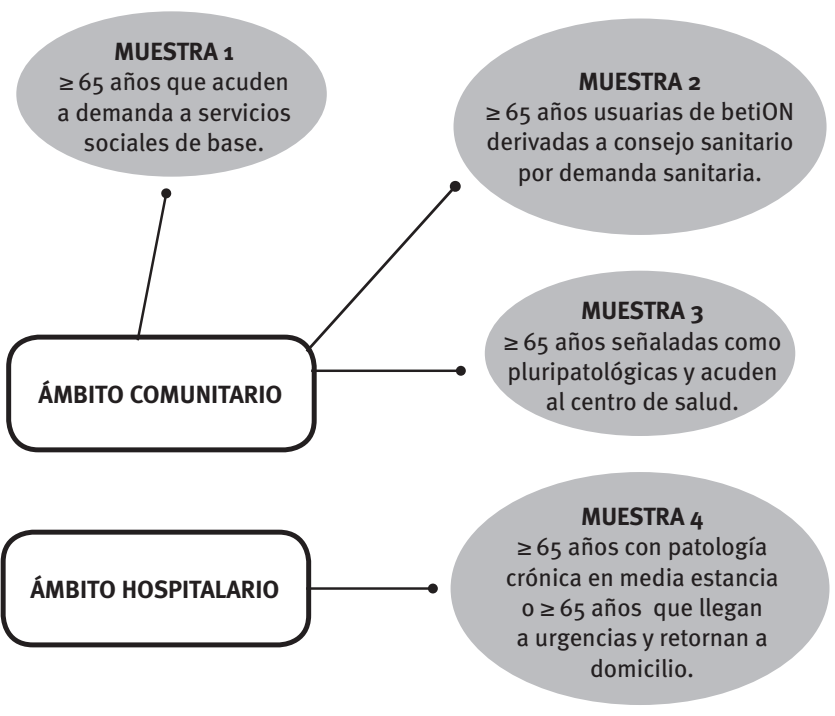

3.

Grupos de discusión tras pilotaje 


\subsection{Análisis cualitativo mediante grupos de discusión}

\subsubsection{Objetivos y metodología}

Los resultados obtenidos de la aplicación de los cuestionarios constituyeron el material de referencia para realizar posteriormente un estudio de tipo cualitativo, basado en la técnica de grupos de discusión. Los y las profesionales debatieron en los grupos sobre la utilidad de la herramienta, comentaron su experiencia a la hora de aplicar el cuestionario y analizaron los resultados alcanzados. Los objetivos de este análisis cualitativo fueron:

- Conocer la opinión de los y las participantes en la experiencia piloto sobre la propia prueba.

- Averiguar su parecer sobre la formación recibida para realizar la prueba piloto (organización, sesiones, duración).

- Conocer sus propuestas para mejorar el periodo formativo.

- Detectar su disposición a adoptar una herramienta de las características de la interRAI CA en su trabajo cotidiano, recogiendo también su opinión sobre la actitud del sector profesional sociosanitario al respecto.

- Determinar su evaluación de la propia herramienta interRAI CA, desde una perspectiva general, como instrumento o herramienta de cribado.

- Describir cómo evalúan las características de la herramienta interRAI CA (validez, fiabilidad, consistencia, sensibilidad).

- Establecer los puntos fuertes y débiles de la herramienta interRAI CA como elemento de cribado.

- Discutir, analizar y establecer el potencial de implantación de una herramienta como la interRAI CA y describir las perspectivas de futuro de las personas participantes al respecto.

- Examinar la situación sociosanitaria actual en nuestro contexto y evaluar la idoneidad de introducir una herramienta de cribado como la interRAI CA en los servicios comunitarios y hospitalarios de la CAPV.

- Evidenciar los frenos y obstáculos que las y los profesionales del sector detectan a la hora de implantar una herramienta de esas características.

- Establecer los requisitos (cambios) que deben producirse en nuestro entorno sociosanitario para el éxito de una herramienta de cribado como la interRAI CA.

\subsubsection{Principales resultados de la fase cualitativa}

El Cuadro 3 recoge, de forma sintética, los aspectos positivos y negativos mayoritariamente reseñados por las personas participantes en los grupos de discusión en relación a la herramienta analizada, según su experiencia durante la prueba piloto.
De los resultados de esta fase de la investigación, cabe subrayar la existencia, entre los y las profesionales participantes, de un discurso maduro, homogéneo y compartido sobre esta cuestión. En concreto, reclaman avanzar en el abordaje sociosanitario integral y en dar una respuesta conjunta, mediante equipos sociosanitarios que compartan espacios de trabajo y recursos, a las necesidades detectadas en las valoraciones integrales de las personas.

Por lo que se refiere a las propiedades intrínsecas de la herramienta, consideran, en general, que se trata de una herramienta válida, que puede ser valorada entre el aprobado alto y el notable. Los y las profesionales consultados también coinciden, sin embargo, en que se trata únicamente de una herramienta, que será eficiente, aumentará la comunicación entre los ámbitos social y sanitario, y mejorará la calidad de la asistencia sociosanitaria en la medida que forme parte de cambios estructurales de mayor calado. En este sentido, opinan que una herramienta, por sí sola, no puede cambiar la filosofía de la atención sociosanitaria, y que es preciso también que las organizaciones cambien, maduren, evolucionen y orienten su trabajo de forma que se eviten duplicidades y se favorezcan el trabajo trasdisciplinar e interdisciplinar. A juicio de estos/as profesionales, en cualquier caso, haber iniciado una experiencia piloto de estas características, en la que están involucradas todas las instituciones, puede interpretarse ya como un rayo de esperanza.

\begin{tabular}{|l|l|}
\hline \multicolumn{2}{|c|}{ Cuadro 3. Ventajas y desventajas atribuidas a la } \\
herramienta en los grupos de discusión \\
\hline \multicolumn{1}{|c|}{ Ventaj } & \multicolumn{1}{c|}{ Desventajas } \\
\hline - Es fácil, rápida y sencilla. & - Le sobra información \\
- Recoge la opinión de usuarios/as & sanitaria. \\
y cuidadores/as. & - Supone una herramienta \\
- Ofrece una foto global, integral & más y, por tanto, más \\
y real. & trabajo. \\
- Es compartido por diferentes & Es sensible al \\
profesionales. & administrador. \\
- Permite la participación de todas & - Le faltan alertas. \\
las personas concernidas. & No ofrece un plan de \\
- Ayuda a la coordinación. & cuidados (?). \\
- Plantea un lenguaje común. & Responde a un modelo \\
- Prioriza necesidades. & anglosajón, no está \\
- Prioriza las urgencias. & adaptada a la realidad \\
- Facilita las reevaluaciones & sociocultural vasca. \\
longitudinales. & No encaja con el contexto \\
- Puede reemplazar a otras & organizativo vasco. \\
herramientas. & No encaja con nuestros \\
- Sitúa a los/as distintos/as profe- & recursos. \\
sionales al mismo nivel. & Las instituciones no \\
- Proporciona informes y explota- & están lo suficientemente \\
ciones estadísticas. & maduras. \\
- Responde a una necesidad del & \\
sector. & \\
- Permite un plan de intervención & \\
conjunta. & \\
& \\
\hline
\end{tabular}

Fuente: Elaboración propia.

Así pues, y de acuerdo con las opiniones manifestadas en los grupos, la introducción de la herramienta analizada debería ir acompañada de otras medidas que generen un entorno en el que ésta pueda mostrar todo su potencial y eficacia. Desde este punto de 
vista, pasar de ofrecer una atención social y otra sanitaria a ofrecer una verdadera atención sociosanitaria requeriría completar el siguiente proceso:

1. Acuerdo y coordinación institucionales.

2. Trabajo en equipos sociosanitarios.

3. Mejora de la herramienta interRAI CA.

4. Sensibilización de los y las profesionales del sector.

5. Formación de los y las profesionales del sector.

6. Definición del perfil de los/as usuarios/as que se han de valorar.

7. Materialización de la historia sociosanitaria.

8. Recursos asistenciales compartidos, y que respondan a las valoraciones y los planes de cuidados.

9. Acceso de todas las partes implicadas a la aplicación informática.

\section{Discusión: avances realizados y retos pendientes}

En los apartados anteriores, se ha dado cuenta del contexto en el que se enmarca el estudio realizado para escoger una herramienta común y consensuada lectura meso de las implicaciones de los resultados obtenidos -frente a la lectura micro que cabe atribuir a las personas participantes en los grupos de discusión y a la visión macro de los decisores públicos a los que se orientan las recomendaciones- y que no coincide necesariamente con la opinión de todas y cada una de las personas consultadas. Dicho de otro modo, para la formulación de las conclusiones y las recomendaciones se ha optado por una lectura estratégica de los resultados obtenidos, tanto en lo que se refiere a las características intrínsecas de la herramienta como a sus posibilidades de implantación en el contexto de la CAPV.

A modo de cierre del artículo, en este apartado se detallan los principales avances derivados del de comunicación entre el sistema de salud y el de servicios sociales.

\subsection{Constatación de que los y las profesionales del ámbito social y de la salud comparten la necesidad de un lenguaje común}

Es necesaria una herramienta, ésta u otra (enfermera de atención primaria).

Es una necesidad, la tenemos en mente (enfermera hospitalaria).

Lo que nos acerque, estupendo (trabajadora social de los servicios sociales de base).

Una primera aportación del proyecto que, lejos de ser baladí, justifica el objetivo general que se pretendía alcanzar y, por ende, el propio trabajo realizado, es que se ha podido confirmar que los y las profesionales del sector social y sanitario, pertenezcan al nivel asistencial al que pertenezcan, comparten la necesidad de adoptar un lenguaje común en el que basar la valoración de las personas con necesidades complejas. Efectivamente, aunque la introducción de un nuevo instrumento de valoración, por excelente que sea, puede encontrarse con reticencias por parte de algunos/as profesionales de ambos sectores, en los grupos de discusión realizados surgió de manera espontánea un discurso homogéneo sobre la necesidad de disponer de herramientas de valoración comunes y compartidas, de trabajar mancomunadamente en el establecimiento de planes de intervención, y de compartir los distintos recursos y servicios ahora compartimentados. Además, se ha constatado la existencia, en todos los niveles de atención implicados, de grupos de profesionales motivados y proactivos que pueden liderar el proceso de cambio si se les ofrecen la oportunidad y los medios para hacerlo.

\subsection{Identificación de una herramienta con los criterios de validez necesarios para constituir el instrumento común de valoración para los servicios sociosanitarios de nivel básico de la CAPV}

mendaciones derivadas del proyecto parten de una proyecto de cara al logro de un espacio compartido
[El interRAI CA] es un instrumento fiable, ya que es consistente en la medición de distintas personas con similares características sociosanitarias, y es sensible, es decir, recoge adecuadamente las variaciones o modificaciones en la situación sociosanitaria de la persona valorada. Además, sirve como puerta única de entrada al ámbito sociosanitario, para estratificar de forma adecuada y fiable, para graduar el nivel de urgencia y para poder implementar un plan de cuidados posterior (miembro del equipo investigador).

El principal objetivo de la investigación consistía en proponer una herramienta susceptible de ser adoptada como puerta de entrada única al sistema sociosanitario, que garantizase el acceso equitativo 
a los recursos y ofreciese orientación en cuanto a la ruta sociosanitaria. A juicio del equipo redactor de este artículo, las características del interRAI CA que hacen de él el instrumento idóneo para ser aplicado en los niveles básicos del sistema sociosanitario en la CAPV son las siguientes:

- Validez, fiabilidad, sensibilidad: como el resto de los instrumentos de la familia RAI, ha sido validado a través de numerosas investigaciones empíricas. Éstas aportan pruebas de que se trata de un instrumento válido, porque sus resultados se ajustan bien a la situación real de la persona; de que es fiable, en el sentido de que sus resultados son consistentes al margen de quién lo aplique; y también de que es sensible, ya que puede detectar cambios en la situación sanitaria y social de la persona.

- Interoperabilidad: pertenece a una familia integrada de instrumentos que permite la trasferencia de datos estandarizados entre diferentes niveles del sistema sociosanitario, garantizando que todos los dispositivos de dicho sistema disponen de un núcleo de información común y relevante para todos y cada uno de ellos. Dado que, en la CAPV, en el nivel de atención especializada ya se utiliza el RAI Screener Plus para la orientación a los recursos sociosanitarios, adoptar el interRAI CA en los niveles básicos garantizará la interoperabilidad entre los diferentes niveles asistenciales.

- Brevedad y sencillez: es un instrumento lo suficientemente breve y sencillo para poder aplicarse en los niveles de atención primaria, lo que le otorga las características necesarias para funcionar como puerta de entrada al sistema de atención sociosanitaria en la CAPV.

- Capacidad de cribado: a pesar de su sencillez, el interRAI CA recoge la información esencial que se necesita en el momento de la demanda de servicios para apoyar la decisión sobre la necesidad de una valoración integral (en la que se puede utilizar el RAI Screener Plus o el Home Care), la necesidad urgente de apoyo en el domicilio y la necesidad de servicios especializados (por ejemplo, de rehabilitación). Esto permitirá a los y las profesionales de atención primaria de salud, de los servicios sociales de base municipales y de los servicios de urgencias hospitalarias detectar con cierta rapidez, y de una manera sencilla, a aquellas personas que se beneficiarían de una valoración en profundidad de sus necesidades sanitarias o sociales, y derivarlas al servicio correspondiente con la urgencia requerida. Además, gracias a la utilización de un mismo lenguaje y de una plataforma informática común, los y las profesionales del sistema al que fuera derivado el caso podrían responder de inmediato a la demanda de atención. Por último, un adecuado cribado de la población susceptible de requerir una valoración en profundidad de sus necesidades también redundaría en una mayor eficiencia del sistema, al evitar aplicar instrumentos más complejos -y, por lo tanto, de mayor coste- en casos en los que posteriormente se determina que la persona no requiere servicios.

- Objetividad en la asignación de servicios: si bien el interRAI CA no genera protocolos de planificación de la asistencia, como ocurre con otros instrumentos de la familia, sí ofrece la información necesaria para planificar a largo plazo servicios e intervenciones en el domicilio, basándose en criterios objetivos de necesidad y urgencia en el acceso.

- Carácter preventivo: el instrumento es sensible al agravamiento de situaciones previamente estables, tanto desde el punto de vista sanitario como social, lo que permite intervenir de manera preventiva, antes de que el estado de la persona empeore.

- Trasdisciplinariedad: el instrumento puede ser aplicado por personas de diferente perfil profesional (médicos/as, enfermeros/as, trabajadores/as sociales) tras un breve periodo de formación ${ }^{3}$. Así, coloca a los diferentes profesionales en el mismo plano para el diagnóstico de las necesidades (valoración trasdisciplinar), lo que permite una intervención interdisciplinar.

- Posibilidad de aplicación telefónica: dada la brevedad del instrumento, no requiere necesariamente aplicarse de forma presencial, y puede hacerse a distancia mediante plataformas telefónicas.

- Posibilidad de explotación estadística para la gestión: la información recogida a través del interRAl CA, además de posibilitar un diagnóstico compartido de las necesidades sociales y sanitarias, y facilitar la elaboración de un plan de intervención compartido, genera, con extremada rapidez y facilidad, datos estadísticos útiles para la gestión a distintos niveles de agregación. Así, resulta posible analizar los datos a escala territorial, pero también por secciones (diferenciando entre atención primaria, consejo sanitario, hospitales, urgencias y servicios sociales de base) e incluso a escala de cada organización (al ofrecer, por ejemplo, los datos del Centro de Salud Amara Berri, del Hospital Txagorritxu o del Servicio Social de Base de Irala-San Adrián).

- Comparabilidad con otros países: puesto que se trata de un instrumento estándar que se aplica en varios países, permite la comparación de las necesidades sociosanitarias existentes en la CAPV con otras realidades de nuestro entorno.

En definitiva, mediante el trabajo realizado se ha identificado un instrumento válido, fiable y ágil, que resulta útil para realizar un primer cribado de la población potencialmente demandante de servicios sociosanitarios. La realización de este cribado tendría, al menos, tres ventajas:

${ }^{3}$ La duración de la formación requerida se estima en torno a dos o tres días de sesiones presenciales, y hasta una semana para las personas con menor experiencia clínica. 
- por una parte, mejoraría la continuidad asistencial para la persona usuaria, agilizando las derivaciones y facilitando el acceso a los recursos adecuados;

- por otra, garantizaría la equidad en el acceso a los servicios para toda la ciudadanía, preservando el principio ético de justicia en la atención;

- y, por último, mejoraría la eficiencia de los dos sistemas de atención, gracias a un mejor ajuste entre las necesidades y los recursos, y a una mejor coordinación de la atención prestada entre ambos.

\subsection{Consenso previo, entre actores de las diferentes instituciones implicadas, para la utilización del instrumento}

Tras la selección del instrumento que, de acuerdo con criterios científico-técnicos, mejor responde a los objetivos generales del proyecto, el propio instrumento y los materiales generados en las fases previas de la investigación (revisión bibliográfica y consenso del grupo científico-técnico de expertos/as) se sometieron a discusión en un grupo de expertos, formado por representantes de las distintas administraciones implicadas en la atención sociosanitaria en la CAPV.

En las sesiones realizadas con este grupo de expertos/as, se alcanzó un consenso para la utilización del interRAI CA tanto en la red sanitaria como en la social. Este consenso constituye un hito importante en el proyecto, ya que en dicho grupo estaban representadas las diferentes instituciones que tendrán que participar en la aplicación del instrumento y que, en cualquier caso, son competentes en la prestación de los servicios sociales y de salud en Euskadi.

\subsection{Adaptación del instrumento}

Como se explicado con anterioridad, en el marco del proceso de investigación, se han traducido, adaptado y validado el cuestionario y el manual de uso al contexto de Euskadi; se ha creado una aplicación informática en castellano, intuitiva, amigable y fácil de usar, que permite administrar el instrumento de una forma sencilla; y se ha puesto a punto la aplicación informática, gracias a la cual se pueden obtener los algoritmos adaptados a la realidad los sistemas social y sanitario de la CAPV. Cabe destacar que la creación de esta aplicación informática es, en sí misma, una primera aportación de interés de este proyecto.

\subsection{Verificación de la utilidad de la herramienta como instrumento común de cribado en nuestro ámbito}

Esto es una primera foto real, que nos puede servir a todos (enfermera hospitalaria).

Me ha resultado útil (trabajadora social de los servicios sociales de base).
La herramienta es fácil, intuitiva (enfermera de atención primaria).

Detecta necesidades (médico de atención primaria).

Unifica lo que hacemos todos, dando un mínimo a todos (médico de atención primaria).

Mira al paciente desde una perspectiva global (enfermera hospitalaria).

Pregunta lo mínimo, saca lo máximo (miembro del equipo investigador).

Tal y como se puso de manifiesto en los grupos de discusión realizados, los y las profesionales de primera línea del ámbito social y sanitario de la CAPV consideran que el interRAI CA reúne la mayoría de los requisitos que se le exigirían a un instrumento de valoración sociosanitaria común, si bien la introducción de algunas modificaciones de menor calado podría incrementar su grado de adaptación a nuestro contexto. En cualquier caso, su aplicación supondría un avance respecto a la situación actual, y cabe pensar que, si no se optara por su implantación, generalizada o parcial, se generaría una cierta frustración en el sector. Éste es el principal resultado obtenido en los grupos de discusión.

De acuerdo a la información recogida en esa fase del estudio, puede afirmarse que la mayoría de los y las profesionales que participaron en la prueba piloto coinciden a la hora de señalar una serie de puntos fuertes de la herramienta:

- Ofrece un lenguaje común con el que manejarse en el diagnóstico de las necesidades de la persona, lo que facilita la coordinación entre el sistema de salud y el de los servicios sociales.

- Brinda una foto global, integral y real de la situación de la persona: el instrumento arroja valoraciones similares para personas en situaciones parecidas; es decir, es capaz de objetivar la situación de la persona.

- Es fácil, rápida y sencilla: se rellena con facilidad y rapidez, lo que resulta primordial para un instrumento de cribado que vaya a utilizarse como puerta de entrada a la red sociosanitaria. Esta valoración positiva se extiende a la aplicación informática utilizada, que resulta amigable y fácil de utilizar.

- Prioriza necesidades: discrimina entre diferentes niveles de urgencia, lo que permite establecer prioridades, basándose en criterios objetivos, a la hora de asignar servicios.

- Incorpora la percepción subjetiva de la propia persona usuaria: siendo un instrumento objetivo para medir las necesidades de las personas, incorpora también la percepción subjetiva de éstas en cuanto a su nivel de bienestar, lo que permite tenerlo en cuenta a la hora de asignar los servicios. 
Con todo, de acuerdo con las opiniones recogidas en los grupos de discusión, el instrumento también presenta algunos puntos débiles e insuficiencias, entre las que cabe destacar las siguientes:

- Escasa consideración de elementos de tipo social: el principal reproche que los y las participantes en la experiencia piloto hacen a la herramienta es su sesgo sanitario, y la comparativamente menor atención que presta a determinados elementos sociales, como los recursos económicos o el perfil de los/as cuidadores/as informales.

- Ausencia de información sobre barreras arquitectónicas en la vivienda de la persona usuaria: la inclusión en el formulario de algún ítem que recoja este problema puede resultar útil de cara a planificar la intervención con la persona usuaria y, de esta manera, mejorar la usabilidad del instrumento en los niveles básicos de atención sociosanitaria.

- Ajustar las salidas del instrumento a los recursos y plazos de atención que se dan actualmente en la CAPV: algunos/as profesionales mencionan que los resultados del instrumento en cuanto a los servicios que precisa la persona y los plazos de urgencia con los que precisa la atención están excesivamente atados a la realidad de los servicios para los que fue creado el instrumento (en este caso, los canadienses) y no se ajustan bien a la situación actual de los servicios sanitarios y, en especial, los servicios sociales de la CAPV. Por ello, consideran que revisar la adaptación de estos resultados a la realidad de los servicios vascos podría incrementar de manera significativa la utilidad del instrumento de cara a su aplicación en nuestro medio.

- Capacidad de cribado: mientras que hay profesionales, sobre todo médicos/as y de enfermería, que creen que mediante la valoración que ofrece la herramienta se pueden detectar necesidades que puedan requerir una ulterior y complementaria valoración, ahondando en ciertos aspectos, otros/as profesionales muestran sus dudas. En todo caso, en la encuesta final la mayoría se decantaba por reconocer su capacidad discriminatoria, de segmentación y de graduación de niveles de urgencia, a pesar de los distintos criterios manejados por los diferentes profesionales.

- Claridad en los diagnósticos: a juicio de algunas de las personas consultadas, la aplicación no tiene bien resuelta la cuestión de los diagnósticos, lo que complica su codificación.

Se señalaron también otros puntos débiles e insuficiencias, como la posible carga adicional de trabajo que supondría la aplicación generalizada y sistemática del instrumento; la excesiva sensibilidad al administrador, con lo que los resultados variarían en exceso según la formación o la extracción profesional de la persona responsable de su aplicación; la falta de alertas; el sesgo cultural anglosajón; la dificultad de adaptar la herramienta al marco competencial y organizacional de la CAPV en lo que se refiere a los servicios sociales y sanitarios; y la inmadurez del propio contexto institucional vasco para la implantación de una herramienta de estas características.

En cualquier caso, el equipo investigador considera que la mayor parte de los obstáculos señalados obedecen, más que a carencias reales o intrínsecas de la herramienta, a circunstancias relacionadas con la fase de formación, con las características de la prueba piloto e, incluso, con las posibilidades de una implementación exitosa en el contexto competencial y organizacional de los servicios sociosanitarios en la CAPV. En ese sentido, se considera que una inadecuada transmisión, durante la fase de formación, de los objetivos y funcionamiento de la herramienta, y más concretamente de su naturaleza de instrumento de cribado, se pudo traducir, en ocasiones, en unas expectativas excesivas o poco ajustadas de lo que podrían obtener de ella. Esto explica que durante los grupos de discusión se recogieran opiniones relativas a la insuficiencia del instrumento para recoger toda la información necesaria para intervenir profesionalmente, o a su incapacidad para sustituir otras herramientas o escalas actualmente utilizadas. También el hecho de que las valoraciones realizadas durante la fase piloto no se completaran con la elaboración de un plan de intervención compartido limitó, en cierta medida, la perspectiva de los y las profesionales en cuanto a las posibilidades del instrumento.

Por otra parte, durante la fase piloto, se produjo cierta indefinición o modificaciones en cuanto al perfil de las personas a las que aplicar el instrumento de valoración, lo que también pudo influir en la percepción de los y las profesionales sobre la adecuación del instrumento.

\subsection{Identificación de las oportunidades y amenazas existentes para la implantación del instrumento en la CAPV}

Una de las conclusiones básicas del estudio es que la percepción sobre las posibilidades de implantación de un instrumento común de valoración para los servicios sociales y sanitarios en la actualidad varían según la perspectiva (macro/meso/micro) desde la que se plantean. En cualquier caso, resulta necesario subrayar que las oportunidades y amenazas detectadas en ningún caso resultan específicas del instrumento interRAI CA, sino que se refieren al contexto social e institucional en el que se debe producir la implantación, por lo que se presentarían invariablemente fuera cual fuera el instrumento escogido para su implantación.

En las perspectivas macro y meso, el equipo investigador y los expertos y expertas institucionales que han debatido las conclusiones del proyecto de investigación coinciden a la hora de señalar la oportunidad que se da en las circunstancias actuales para implantar un instrumento común de valoración que funcione como puerta de entrada a la red de servicios 
sociosanitarios y como lenguaje común de mínimos para construir el modelo de atención sociosanitaria comunitario. Se señala, en este sentido, el alineamiento, en esta dirección, de los respectivos planes estratégicos sectoriales (Osakidetza, 2013; Gobierno Vasco-Eusko Jaurlaritza, 2011), así como de las líneas estratégicas marcadas por el Consejo Vasco de Atención Sociosanitaria (2005, 2010, 2014).

También existe consenso a la hora de calificar de oportunidad la existencia de otros proyectos que enlazan con los objetivos de la investigación desarrollada, reforzándolos. Algunos de los más importantes son:

- El acceso a Osabide Global desde los centros residenciales para personas mayores. Los dos elementos clave en cuanto a la información que cualquier profesional social o sanitario puede requerir de una persona usuaria son la historia clínica y la historia sociosanitaria. Respecto a la primera, se prevé que, para finales de $\mathbf{2 0 1 5}$, todos los centros gerontológicos de más de cien camas tendrán acceso directo a la historia clínica de la persona usuaria, previo consentimiento informado de ésta. Consolidado ya este primer paso hacia una historia clínica compartida, es el momento idóneo, y de ahí su oportunidad, para aunar esfuerzos e implantar el interRAI CA como primer paso hacia lo que será la historia sociosanitaria.

- La incorporación, dentro del Plan de Salud 20132020 (Departamento de Salud, 2013), de valoraciones integrales que se realizarían con carácter preventivo a todas las personas mayores de 65 años con patologías crónicas, y a todas las que superan los 75 años de edad. Este proyecto ofrece la oportunidad de utilizar el interRAI CA para realizar dicha valoración.

- La integración, desde 2011, de la atención social y sanitaria a distancia en el sistema betiON, que supone una oportunidad para la incorporación del interRAI CA como instrumento común de valoración de aquellas personas usuarias de teleasistencia con necesidades complejas sociales y sanitarias.

Desde el nivel micro, en el que se sitúan los y las profesionales de atención directa que han participado en la prueba piloto y en los posteriores grupos de discusión, se destaca también como oportunidad la existencia de una necesidad, compartida por todo el sector, de construir un lenguaje común con el que trabajar, que se deja sentir cada vez más y se hace ineludible al enfrentarse a los nuevos retos sociales, como el envejecimiento, la cronicidad o la atención a la terminalidad, y a una sociedad mucho más exigente en cuanto a la calidad de la atención que se le presta desde los servicios públicos. Cabe destacar asimismo la existencia de un núcleo de profesionales, especialmente concienciados/as y motivados/as, que puede actuar como vector de cambio, ejerciendo el liderazgo para la implantación del proyecto en las distintas organizaciones implicadas.

Pero también se detectan obstáculos y dificultades. Por lo que se refiere a las amenazas para la implantación de un instrumento común de valoración, los y las profesionales de primera línea tienden a considerar que la actual distribución competencial resulta un obstáculo para el trabajo conjunto, y que dificulta la implantación del interRAI CA. A su modo de ver, es difícil que las diferentes instituciones implicadas alcancen un acuerdo que permita implantar el instrumento de manera que resulte útil, sin convertirse en una tarea más que no aporte ningún valor añadido.

Desde el nivel meso y macro, el equipo investigador y el grupo de expertos y expertas institucionales consultados plantean una opinión diferente. Partiendo del hecho de que en la CAPV se ha optado por un modelo de coordinación funcional de dos sistemas autónomos -con sus ventajas y desventajas-, el trabajo debe estar dirigido a establecer puentes entre el sistema sanitario y el de servicios sociales, y es ahí precisamente donde surge la necesidad de un lenguaje común y donde una herramienta como el interRAI CA puede resultar útil. En este sentido, se considera que la actual distribución competencial no constituye un obstáculo para implantar el instrumento; más bien al contrario: es precisamente lo que determina que su implantación sea una necesidad. Esta necesidad, junto con las oportunidades para la implantación a las que se ha aludido, hacen que nos encontremos ante el momento idóneo para dar un impulso definitivo al proyecto.

Desde ese punto de vista, la implantación -progresiva y gradual- del interRAI CA constituiría el núcleo central para la construcción de la historia sociosanitaria en la CAPV, y permitiría un avance sin precedentes en el desarrollo de la interoperabilidad sociosanitaria y en una realidad sociosanitaria basada en el trabajo conjunto de equipos de atención primaria sociosanitarios (EAPSS). 


\section{Bibliografía referenciada}

(2006): “Ley 39/2006, de 14 de diciembre, de Promoción de la Autonomía Personal y Atención a las personas en situación de dependencia", Boletín Oficial del Estado, № 299, 15-12-2006, págs. 44.142-44.156 [<http://www.boe.es/boe/ dias/2006/12/15/pdfs/A44142-44156.pdf)].

CONSEJO VASCO DE ATENCIÓN SOCIOSANITARIA (2014): Líneas estratégicas de atención sociosanitaria para Euskadi 2013-2016.

- (2010): Documento marco para la elaboración de las directrices de la atención sociosanitaria en la Comunidad Autónoma Vasca.

- (2005): Plan Estratégico para el Desarrollo de la Atención Sociosanitaria en el País Vasco 2005 2008, Vitoria-Gasteiz, Servicio Central de Publicaciones del Gobierno Vasco.

DEPARTAMENTO DE SALUD (2013): Osasuna, pertsonen eskubidea, guztion ardura. Políticas de Salud para Euskadi. Plan de Salud 2013-2020 / Osasuna, pertsonen eskubidea, guztion ardura. Euskadirako Osasun Politikak. Osasun Plana 2013-2020, Vitoria-Gasteiz, Eusko JaurlaritzaGobierno Vasco.

ELÓSEGUI, E. et al. (2014): Interoperabilidad de los sistemas de información sociosanitarios. Selección de un instrumento consensuado de valoración sociosanitaria, Barakaldo, BIOEF-Fundación Vasca de Innovación e Investigación Sanitarias [<http://www.siis.net/valoracionsociosanitaria. pdf>].
GOBIERNO VASCO-EUSKO JAURLARITZA (2011): Plan Estratégico de Servicios Sociales de la CAPV 2011-2014, Vitoria-Gasteiz, Servicio Central de Publicaciones del Gobierno Vasco.

GOBIERNO VASCO-EUSKO JAURLARITZA et al. (2003): Convenio de colaboración entre el Gobierno Vasco, las diputación forales de Álava, Bizkaia y Gipuzkoa y la Asociacion de Municipios Vascos Eudel para el desarrollo de atención sociosanitaria en la Comunidad Autónoma del País Vasco [<http://bit.ly/17QvaNu〉].

KODNER, D.; SPREEUWENBERG, C. (2002): "Integrated care: Logic, meaning, implications and applications", International Journal of Integrated Care, vol. 2, noviembre, págs. 1-6.

OSAKIDETZA (2013): Líneas estratégicas y planes de acción. Osakidetza 2013-2016, Vitoria-Gasteiz, Osakidetza.

SIIS CENTRO DE DOCUMENTACIÓN Y ESTUDIOS (2008): Atención sociosanitaria: una aproximación al marco conceptual y a los avances internacionales y autonómicos / Arreta soziosanitarioa: esparru kontzeptuala eta nazioarteko eta autonomietako aurrerapausoak. Hurbilketa, serie: Informes Extraordinarios / Txosten Bereziak, Vitoria-Gasteiz, Ararteko.

URRUTIA, J. M. (2006): "Modelo de valoración de la dependencia unificado para Euskadi”, Osasunaz, nํㅜ 7, págs. 19-36. 
